\section{Addendum: Active superelasticity in three-dimensional epithelia of controlled shape}

https://doi.org/10.1038/s41586-021-03281-0

Addendum to: Nature https://doi.org/10.1038/s41586-018-0671-4

Published online 31 October 2018

Check for updates

Ernest Latorre, Sohan Kale, Laura Casares, Manuel Gómez-González, Marina Uroz, Léo Valon, Roshna V. Nair, Elena Garreta,

Nuria Montserrat, Aránzazu del Campo, Benoit Ladoux, Marino Arroyo \& Xavier Trepat

In our original Article, we developed a phototriggerable derivative of the actin disruptor cytochalasin D, denoted photo cytoD or Nvoc-CytoD, to show that a local perturbation of the actin cortex increases cell area (Extended Data Fig. 8 of the original Article). Through direct visualization, we showed that the F-actin cytoskeleton of cells that were incubated with Nvoc-CytoD $(50 \mu \mathrm{M})$ was rapidly disrupted upon light exposure (Extended Data Fig. 8c). Cells incubated with Nvoc-CytoD without light exposure, and cells exposed to light without Nvoc-CytoD, did not show changes in their cytoskeleton or in cell area (Extended Data Fig. 8a-d). A detailed characterization of the synthesis and chemical validation of Nvoc-CytoD was not included in our original Article, and we would like to provide it here.

Nvoc-CytoD includes a nitroveratryloxycarbonyl (Nvoc) photoremovable protecting group at the hydroxyl group at $\mathrm{C} 7$ of CytoD (Fig. 1a of this Addendum), which is relevant for binding to actin. The attachment of the photoremovable protecting group is expected to inhibit binding of Nvoc-CytoD to actin temporarily, and enable light-dependent delivery of the active drug CytoD to living cells.

The Supplementary Information to this Addendum contains information on the molecular design of Nvoc-CytoD, as well as its synthesis, structural characterization and photochemical properties.

Supplementary information is available in the online version of this Amendment.

1. Nair, U. B., Joel, P. B., Wan, Q., Lowey, S., Rould, M. A. \& Trybus, K. M. Crystal structures of monomeric actin bound to cytochalasin D. J. Mol. Biol. 384, 848-864 (2008).

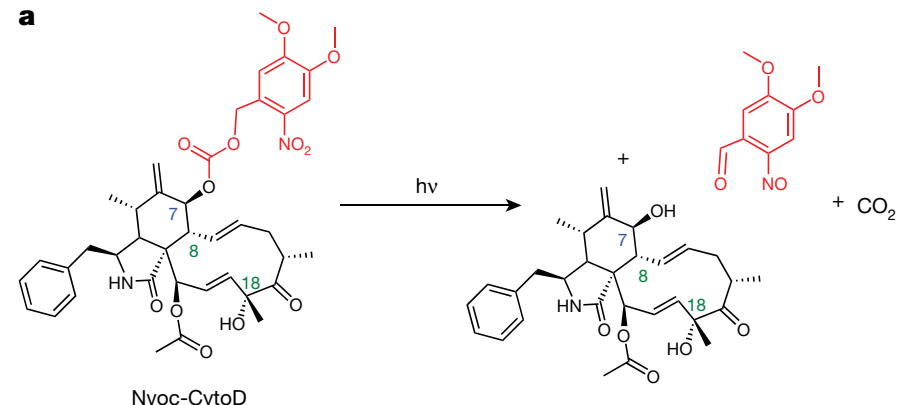

Nvoc-CytoD b

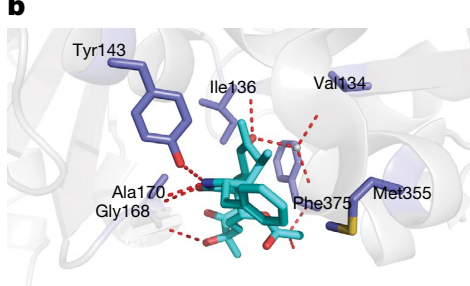

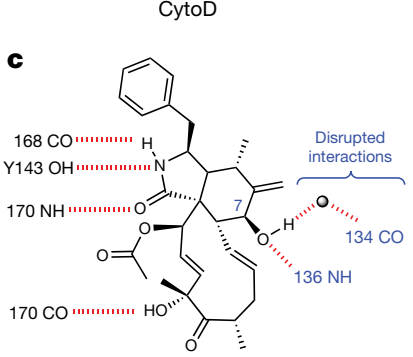

c
Fig. 1 The structure of Nvoc-CytoD and the binding of CytoD to actin. a, Structure of Nvoc-CytoD and its photochemical activation reaction. b, Expanded view of CytoD (cyan sticks) bound to actin monomer (grey). The red dotted lines depict hydrogen-bonding interactions. This figure is recreated from Protein Data Bank (PDB) ID 3EKU (ref. ${ }^{1}$ ) and is rendered using PyMOL. c, The hydrogen-bonding interactions involved in CytoD-actin binding are shown as red dotted lines. Note that the hydroxyl at $\mathrm{C} 7$ interacts with Ile136(NH) and V134(CO). 